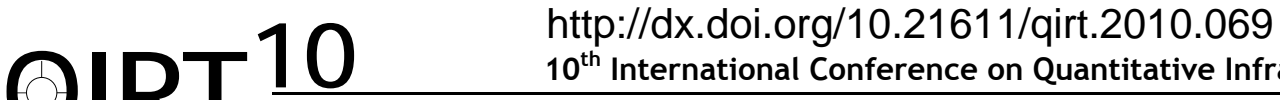 \\ $10^{\text {th }}$ International Conference on Quantitative InfraRed Thermography \\ July 27-30, 2010, Québec (Canada)
}

\section{Calculation and infrared measurement identification of shell temperature distribution on overhead heat pipeline placed in open air space}

by T. Kruczek* and A. Fic*

\author{
*Institute of Thermal Technology, Silesian University of Technology, Konarskiego 22, 44-100 Gliwice, Poland, \\ tadeusz.kruczek@polsl.pl,adam.fic@polsl.pl
}

\begin{abstract}
On the external shell of the heat pipeline convective and radiative heat transfer takes place between pipeline and its surrounding. In the case of low temperature of the sky, the radiation heat exchange is very intensive and the temperature of the top part of the horizontal pipeline is much lower than the bottom part. This phenomenon has been confirmed by measurements and calculations carried out by means of software CFD Fluent. The aforementioned phenomenon causes difficulties during evaluation of the quality of the pipeline thermal insulation and determination of the pipeline heat losses. It is possible to take into account all measurement circumstances and make necessary corrections of measurement results in order to draw proper conclusions concerning the quality of pipeline thermal insulation. An important problem is the proper determination of apparent temperature of the hypothetical sky surface. This temperature influences significantly pipeline external shell temperature and radiative heat losses into environment.
\end{abstract}

\section{Denotations and subscripts}

\begin{tabular}{|c|c|c|c|}
\hline Denotations & & Subscripts & \\
\hline$T$ & temperature, $\mathrm{K}$ & $a$ & atmospheric air \\
\hline$A$ & absorptivity & $S k$ & sky \\
\hline$\varepsilon$ & - emissivity & $\lambda$ & spectral \\
\hline
\end{tabular}

\section{Introduction and aim of the work}

Infrared thermography examination of thermal insulation of pipelines is a very effective and modern measurement method. The result of this measurement is the distribution of temperature on the external shell of pipeline. The knowledge of shell temperature (the temperature inside the pipeline is usually known) allows to evaluate the quality of pipeline thermal insulation. Unfortunately, the result of the temperature thermovision measurement is influenced by many parameters, especially by emissivity of the examined surface and the temperature of the surrounding elements. Convective and radiative heat transfer takes place between thermal pipelines and its surrounding [3, 4], figure 1.
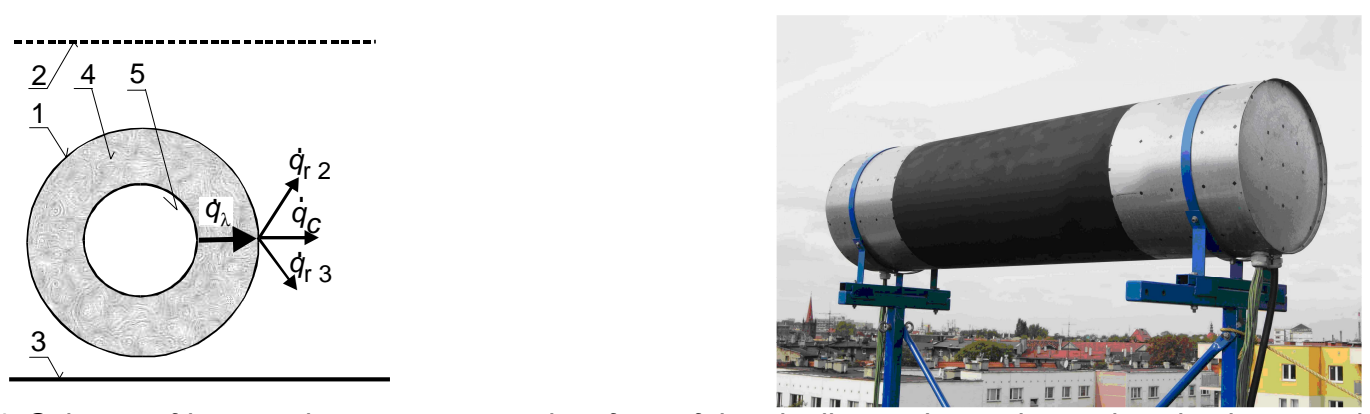

Fig. 1. Scheme of heat exchange on external surface of the pipeline and experimental station in open air space; 1-external shell, 2-sky, 3-bottom, 4-thermal insulation, 5-fluid inside the pipeline

Generally, the surrounding of the external pipeline consists of two surfaces: hypothetical sky surface and bottom surface. Normally, the temperatures of these elements are different. In the case of low temperature of the sky, due to very intensive radiative emission of the heat, the temperature of some parts of the pipeline shell may drop below the temperature of the atmosphere $[3,4]$. Examples of such phenomena may be seen in figure 2. 
Temperature values in several points are printed near the crosses which can be found on the external surface of the pipelines, figure 2. It can be noticed that the temperature of the shell on the upper part of the pipelines is lower than the temperature of atmospheric air. This phenomenon causes some difficulties in analysing the measurement results as well as in proper determination of heat losses. Determination of the heat losses makes it possible to evaluate the quality and technical state of thermal insulation of the pipeline.

The goal of the work was the identification of heat exchange process on the external shell in terms of applying the infrared thermography measurement results in the procedure for the determination of the aforementioned heat losses.

a)

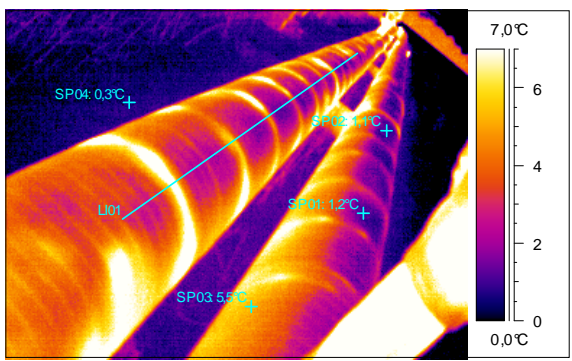

b)

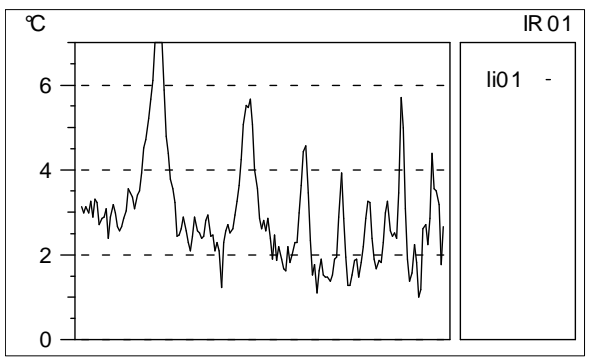

Fig. 2. Sample of measurement results of infrared thermography examination of heat pipeline, $T_{a}=4.5^{\circ} \mathrm{C}$, $\boldsymbol{T}_{\boldsymbol{S} \boldsymbol{k}}=-9.6^{\circ} \mathrm{C}$; a) thermogram, b) temperature profile along the line $\mathrm{L} 101$

\section{Measurements in natural environment with use of experimental unit}

For the research of the heat exchange process on outer surface of pipeline shell the practical measurements in natural environment have been carried out, figure 1. During these experiments the following parameters were measured: the wall temperature of electrically heated pipeline tube, the temperature of external shell of insulation and air temperature by means of thermocouples. Additionally, air humidity, wind direction and velocity, the output of electrical heater were measured. All these quantities were recorded. The temperatures of ambient elements (ground and sky) were measured periodically with -use of infrared camera of LW type ThermaCAM SC2000 (spectral range $7.5 \mu \mathrm{m} \div 13 \mu \mathrm{m}$ ).

As the results of these measurements the distributions of the temperature around the pipeline shell and air temperature have been obtained. The samples of results for different measurement conditions are presented in figures 3,4 , 5 , 6 . These measurements have been carried out during the night periods, usually between $10 \mathrm{pm}$ and $3 \mathrm{am}$.
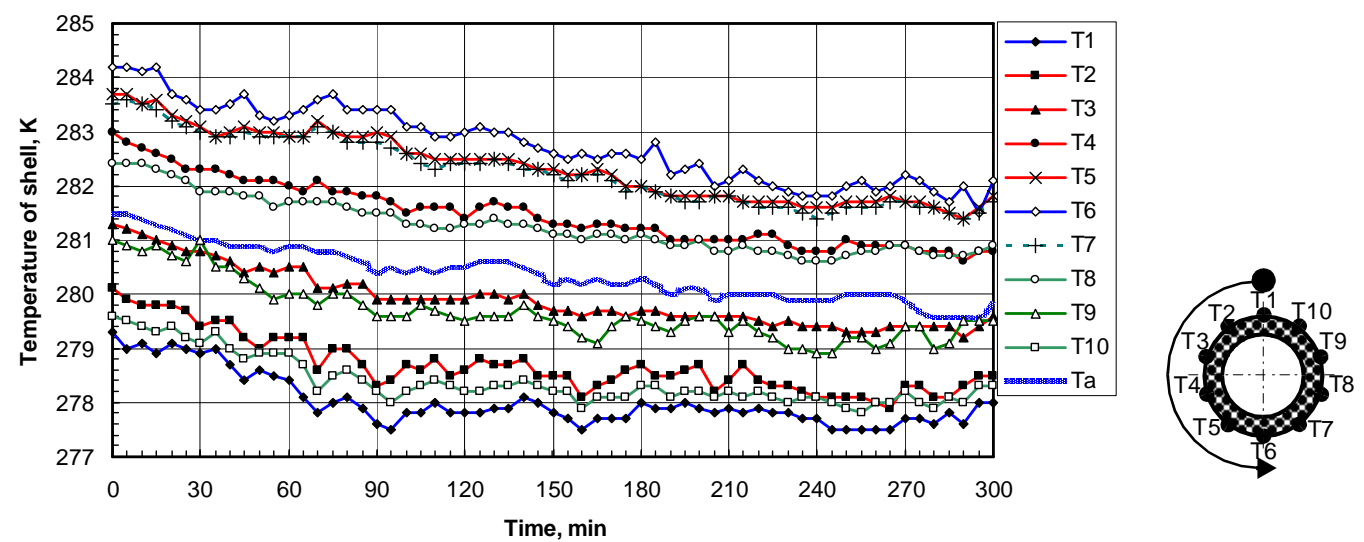

Fig. 3. Distribution of the pipeline outer shell temperature for high emissivity of shell and low temperature of sky, on the right - start point and direction of results collection around the pipeline shell, $\boldsymbol{T}_{\mathbf{a}}$ - atmospheric air temperature

Temperature distribution of the external shell of the overhead pipeline is significantly influenced by low temperature of the sky. These diagrams show that in the case of high emissivity of external pipeline shell and low temperature of sky the temperature of shell may drop below the temperature of atmospheric air, see figure 3 . Usually, at low emissivity of shell its temperature around the pipeline is higher than the temperature of atmospheric air, see figures 5,6 . This phenomenon has been confirmed by numerical calculations. 

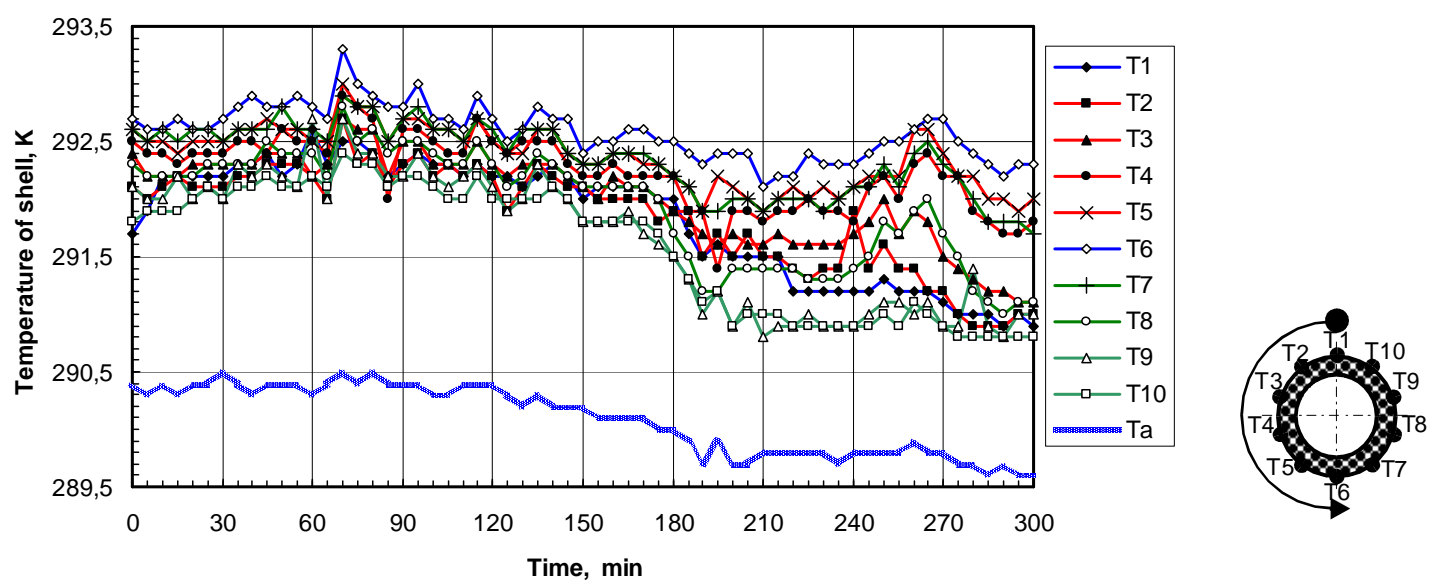

Fig. 4. Distribution of the pipeline outer shell temperature for high emissivity of shell and relatively high temperature of sky, on the right - start point and direction of results collection around the pipeline shell
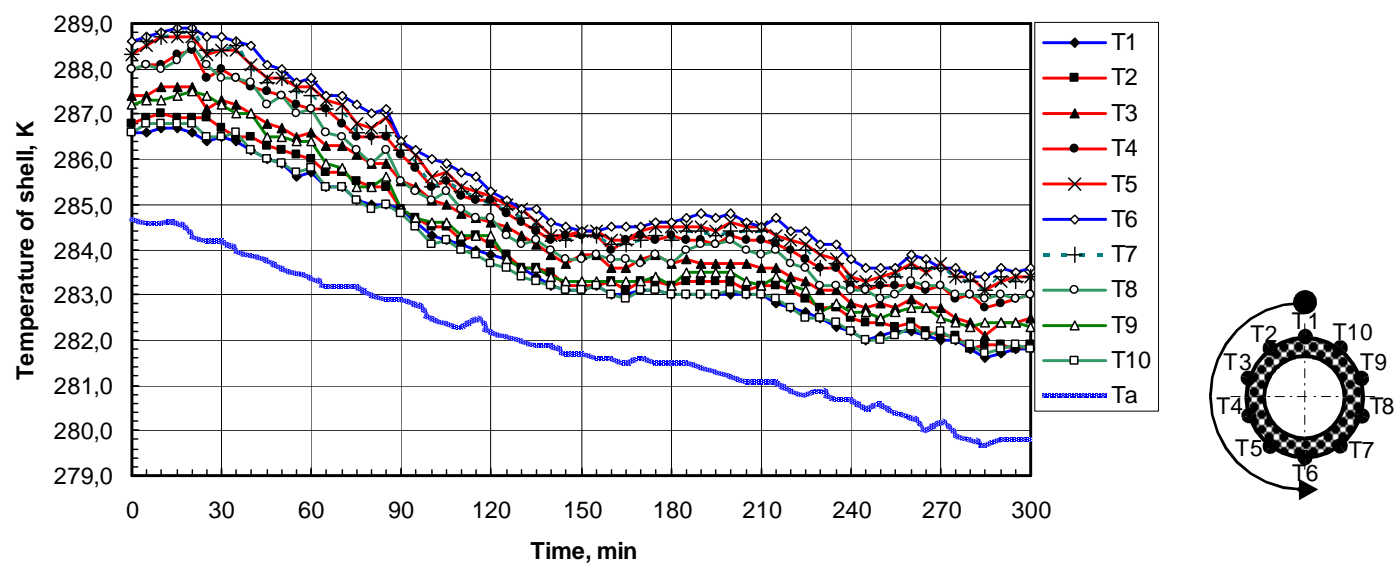

Fig. 5. Distribution of the pipeline outer shell temperature for low emissivity of shell and low temperature of sky
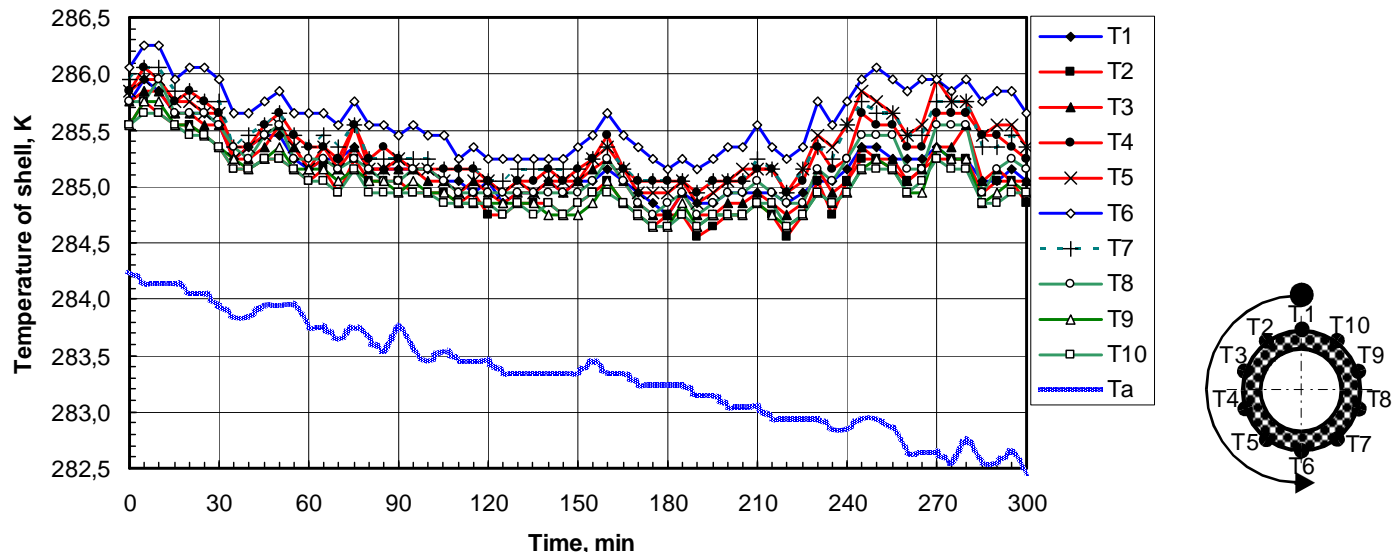

Fig. 6. Distribution of the pipeline outer shell temperature for low emissivity of shell and high temperature of sky 


\section{Application of CFD Fluent software for the analysis of heat exchange between the overhead pipeline and the surrounding}

The packages of CFD (Computational Fluid Dynamics) make it possible to carry out numerical analysis or simulation of processes connected with fluid flow, heat flow processes and other processes [1]. This is achieved by the numerical solution of equations describing these phenomena. The package Fluent version 6.3 was used to analyse the processes occurring on the external surface of the pipeline.

During the creation of the geometrical model, the following components have been distinguished: hypothetical surface of the sky divided into isothermal parts, isothermal surface of the ground, a tube with hot fluid inside, thermal insulation, shell covering the thermal insulation. The geometrical model 2D has been applied at this stage of analysis. The horizontal pipeline was located in the middle of the space of $9 \mathrm{~m}$ in height and $14 \mathrm{~m}$ in width, figure 7 . Air with uniform and determined velocity flows into this space. As a result of calculations, velocity field and air temperature field, as well as the shell temperature, radiative and convective heat fluxes distributions around the pipeline have been obtained.

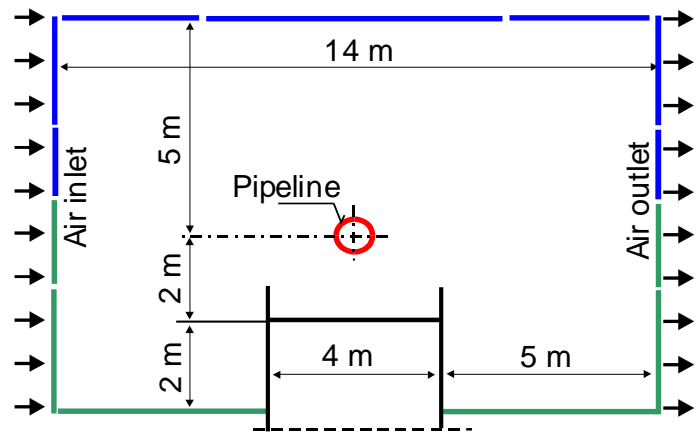

Fig. 7. Scheme of pipeline ambient assumed for numerical calculations

\section{Radiative properties of atmosphere in context of pipeline heat losses determination}

Generally, the ambient of the external overhead pipeline consists of hypothetical sky surface and ground surface. The method concerning the determination of apparent temperature of the hypothetical sky surface has been developed. In this method the radiation of atmosphere towards the ground surface is substituted by the radiation of the assumed artificial surface [3].

The main components of Earth's atmosphere responsible for absorbing or emitting thermal radiation are water vapour and carbon dioxide. To determine the radiative properties of the atmosphere the numerical calculations have been made with the use of data base HITEMP being used for calculation of thermal properties of different gases (similar to HITRAN, LOWTRAN, etc bases). In the analysed spectral range of infrared radiation over $10^{6}$ spectral lines were taken into consideration. The goal of these calculations was the determination of spectral absorptivity of the atmosphere at given thermal parameters of the atmospheric air [2,6,7]. Next, the justifiable assumption has been made that absorptivity $\boldsymbol{A}$ of atmospheric air is equal to its emissivity $\boldsymbol{\varepsilon}$. The results obtained for different thickness of air layers are shown in figure 8 .

Analysing these results we can find that there are distinct windows and radiation bands in the analysed spectral range. For the distinguished bands emissivity (absorptivity) is practically always equal 1.0 and only at smaller thickness of layer the differences in emission bands width are visible, figures $8 \mathrm{a}, \mathrm{b}$. The differences between diagrams in figure $8 \mathrm{c}$ and figure $8 \mathrm{~d}$ are not visible. It means that for the relatively thick layer of atmospheric air over the ground surface these bands may be treated approximately as a black body from the radiation point of view. It is of great importance considering radiative heat exchange between an overhead pipeline and the atmosphere. It means that in the spectral range of bands the heat exchange occurs between pipeline shell surface and the radiatively black body of known temperature. This temperature is the atmospheric air temperature. In the horizontal direction the air temperature is uniform so the assumption of isothermal air layer is justifiable. But in the vertical direction there is a temperature drop of atmospheric air. This drop is average $0.5 \mathrm{~K} \div 0.8 \mathrm{~K}$ at each $100 \mathrm{~m}$ of air layer (height above the earth surface) $[5,8]$.

The influence of atmospheric air temperature drop on its radiation properties was examined by the calculation of absorptivity of isothermic air layer $700 \mathrm{~m}$ thick considering temperature drop for this height. The results were presented in figure 9. The differences are insignificantly tiny. For example, in the spectral range of camera operation equal $7.5 \div 13.0 \mu \mathrm{m}$, after assuming the above mentioned temperature drop the absorptivity (emissivity) changed from 0,172 to 0,170 but in the range of active band $5.4 \div 7.5 \mu \mathrm{m}$ it changed from 0.989 to 0.988 . Likewise is in the case of other windows and radiation bands. 
Summing-up, the air temperature changes in the expected range do not influence significantly the absorptivity (emissivity) of air layer and at the present stage of the analysis and air layer thickness amounting to several hundred meters this effect may be ignored.

a)

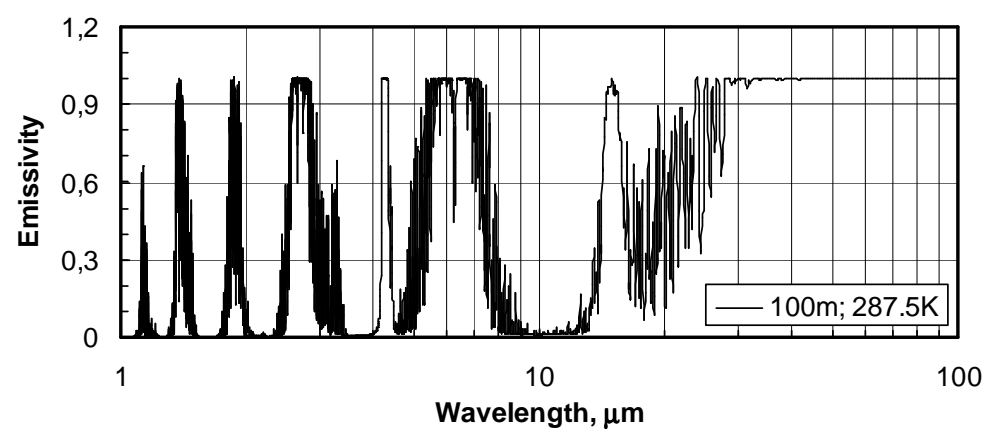

b)

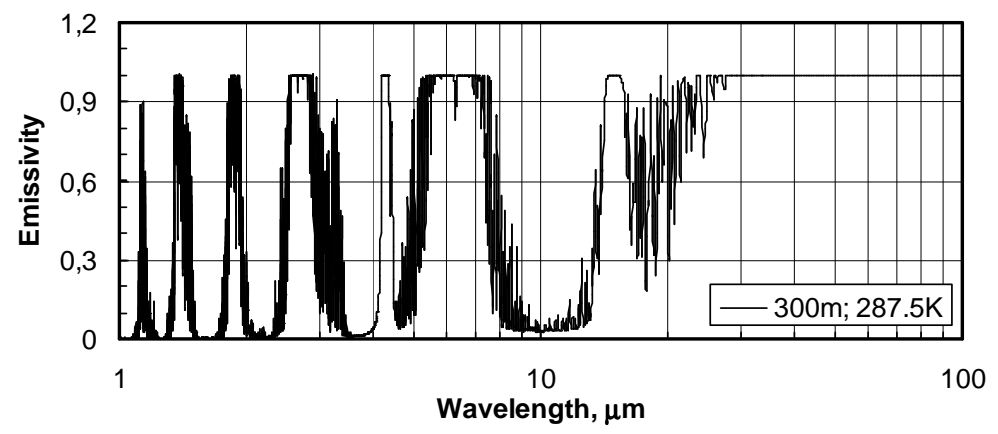

c)

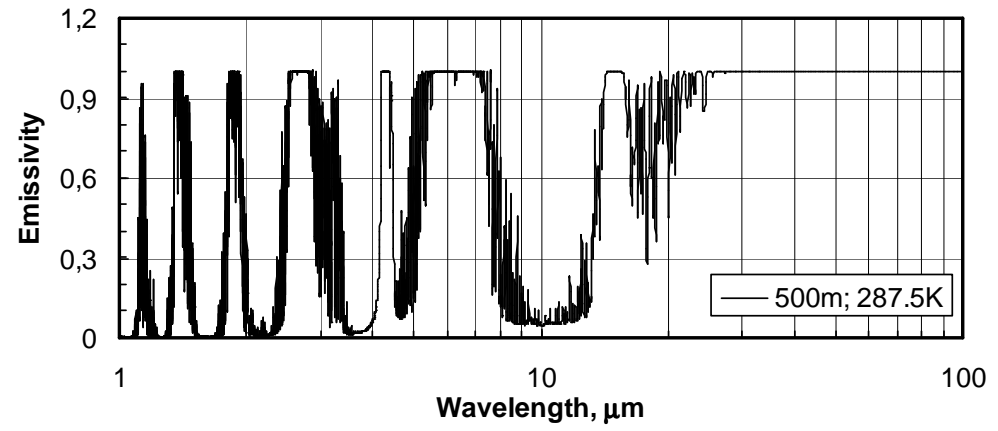

d)

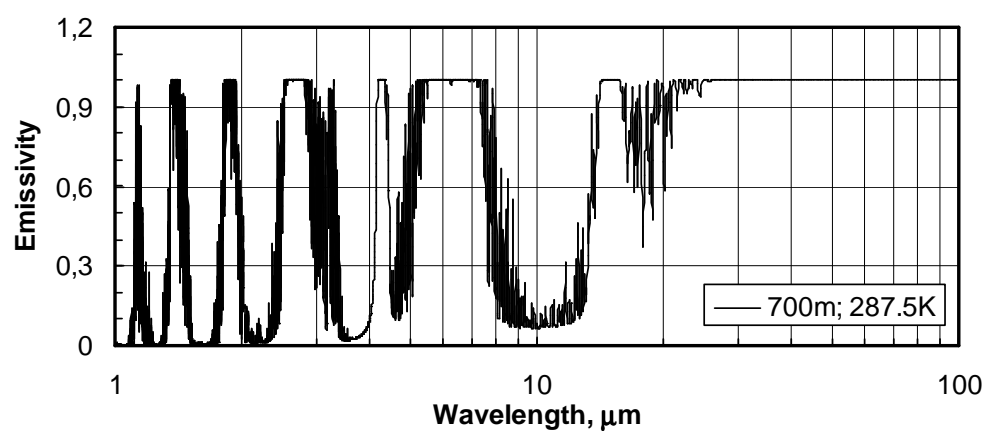

Fig. 8. Emissivity (and simultaneously absorptivity) of atmospheric air layer containing $385 \mathrm{ppm} \mathrm{CO}_{2}$ and $1,1 \%$ of water vapour for temperature and layer thickness specified in the legend 


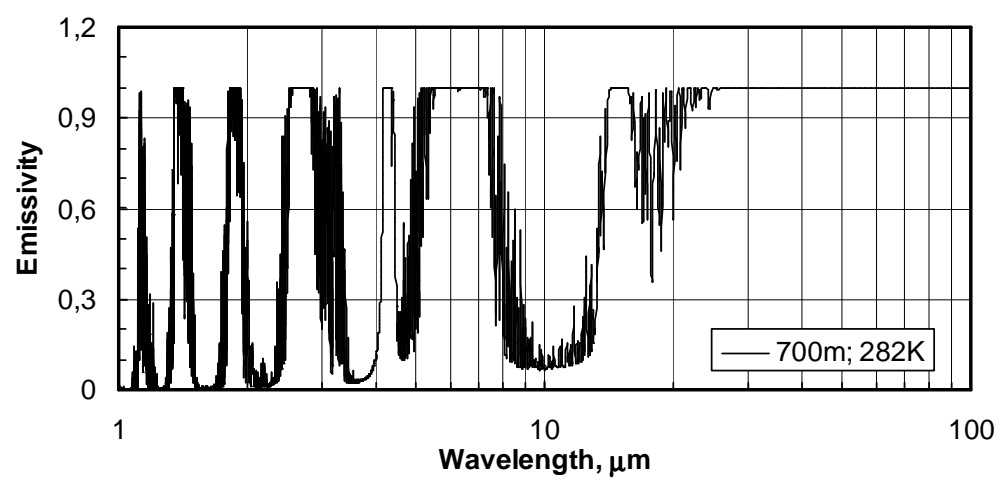

Fig. 9. Emissivity (and simultaneously absorptivity) of atmospheric air layer containing $385 \mathrm{ppm} \mathrm{CO}_{2}$ and $1,1 \%$ of water vapour for temperature and layer thickness specified in the legend

\section{Radiation heat exchange between the pipeline and atmospheric air}

Convective and radiative heat transfer takes place between thermal pipelines and the environment. The surrounding of the external pipeline consists of two elements: hypothetical sky surface and ground surface [4]. Normally, the temperatures of these elements are different. In the case of low temperature of the sky, due to very intensive radiative emission of the heat, the temperature of upper parts of the pipeline shell may drop below the temperature of the atmosphere [4]. Mathematical description of the radiation heat exchange between the pipeline and ground is relatively simple. Some problems may cause the modelling of radiation heat transfer between the pipeline shell and hypothetical sky surface (atmospheric air). The analysis of diagrams in figures 10 and 11 indicates that heat exchange between the pipeline and atmosphere is of bandwise character.

We can assume that the diagram in figure 10 shows the spectrum of radiation intensity of air layer and the diagram in figure 11 shows radiation spectrum of solid black body surface. The radiative heat exchange between the surface of pipeline shell and atmospheric air in the band $5 \div 7.5 \mu \mathrm{m}$ is slightly intensive due to near-values of temperature and high emissivity (absorptivity) of air layer. Considering this high emissivity the layer of air radiates relatively intensively to the surface of pipeline. The similar situation is for the bands of wavelength over $13 \mu \mathrm{m}$, figure 10 . The result is that the difference between energy radiated by the surface of pipeline and energy received from atmosphere (heat loss of pipeline) is relatively small for these bands.

The situation is different for window $7.5 \div 13.0 \mu \mathrm{m}(14 \mu \mathrm{m})$. The energy flux emission from pipeline shell is big because in that spectral range for the typical values of shell temperatures there is the maximum value of spectral density of emission, see diagram in figure 11. At the same time the heat flux emitted by air in window space is small, figure 10 . The result is that the difference between the energy emitted by the surface of pipeline and this received from atmosphere is big which is the main reason of radiative heat losses.

Various models of gaseous layer can be applied to calculate the radiative heat loss of a pipeline in the open space. The model of spectral lines can be applied there which is time-consuming as far as computer calculations are concerned. The models of narrow or wide bands and windows can be applied according to figures 8, 9, 10 [2,6, 7]. At last, the radiation of gaseous layer can be replaced by the radiation of the hypothetical surfaces of temperature that ensures the same intensity of heat exchange as in the case of gaseous layer [3]. 


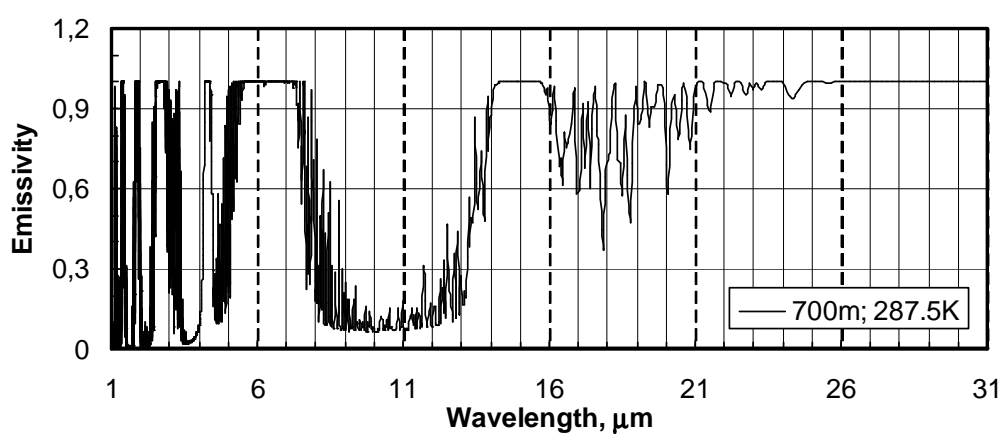

Fig. 10. Diagram showing the absorptivity (emissivity) of atmospheric air for the same parameters as in figure $8 d$ but with uniform wavelength axis

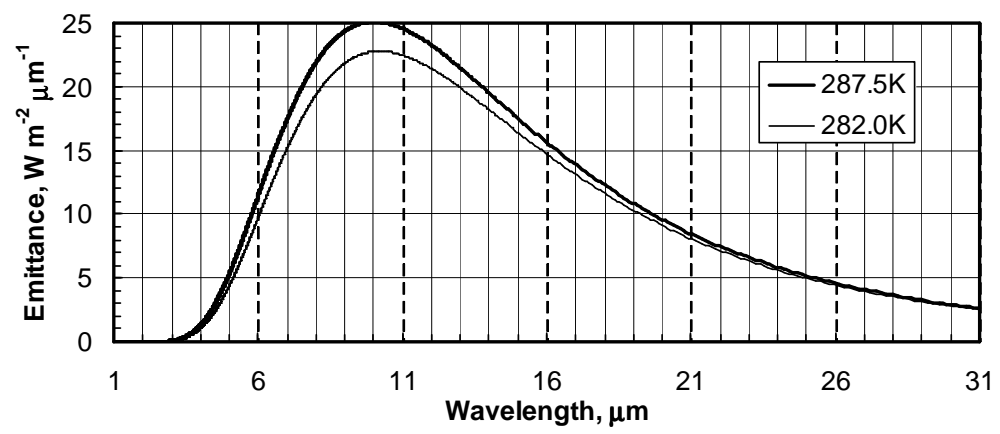

Fig. 11. Distribution of spectral density of black body emission as a function of wavelength

\section{Recommendations and calculation sample}

In order to examine the problem, numerical calculations have been carried out. Figure 12 presents the calculated distributions of the shell temperature around the pipeline. Strong diversification of the shell temperature between the upper and bottom part of the pipeline can be observed in this case. In order to calculate properly the heat losses in this case or evaluate the quality of thermal insulation, this phenomenon should be taken into consideration

Basing on the results we have obtained hitherto we may formulate the following recommendations concerning the calculations of radiation heat losses from overhead pipelines. The radiative heat exchange between the pipeline and atmosphere is of bandwise character. The radiative heat exchange is of small intensity within the band ranges of high absorptivity (emissivity) because of a small temperature difference between a pipeline shell and the air. Considering high absorptivity (and emissivity) of a layer of atmospheric air even with small thickness of this layer, in the radiation heat exchange calculations the air temperature from pipeline vicinity can be assumed.

The radiative heat losses are generated by heat exchange in the spectral window $7.5 \div 14 \mu \mathrm{m}$, see figure 10 . The calculations referring to radiation heat exchange in this window are proposed to be carried out basing on the result of sky radiative temperature (apparent temperature) measurement done by means of camera LW $7.5 \div 13 \mu \mathrm{m}$ or $8 \div 14 \mu \mathrm{m}$, that is the camera we usually use during the pipeline inspections. The infrared camera measurement of apparent temperature of the sky should be carried out with the adjusted distance of an object from a camera equals zero and object emissivity equals 1.0. Therefore the measured temperature will correspond to radiation heat flux which reaches the examined surface from the space (from atmosphere).

There are various possibilities of further calculations. The mathematical model can be applied to calculate mean substitute temperature for the whole spectral range or a band model can be applied in radiation heat exchange calculations $[2,7]$. The differentiation of sky temperature should be taken into consideration according to the observation angle of camera and proper geometry of the analysed system.

In figure 12, the results of exemplary measurements at the real testing unit are presented and also calculation results where the abovementioned recommendations are considered. The calculations are carried out for the case when 
radiation heat exchange has been ignored (case a), for the case when a pipeline is located in entirely open hemispherical space (b), and when in the vicinity of pipeline there are certain elements of the building roof where experimental unit has been placed (c) and this situation has taken place in reality. The consistency of calculation and measurement results has been achieved.

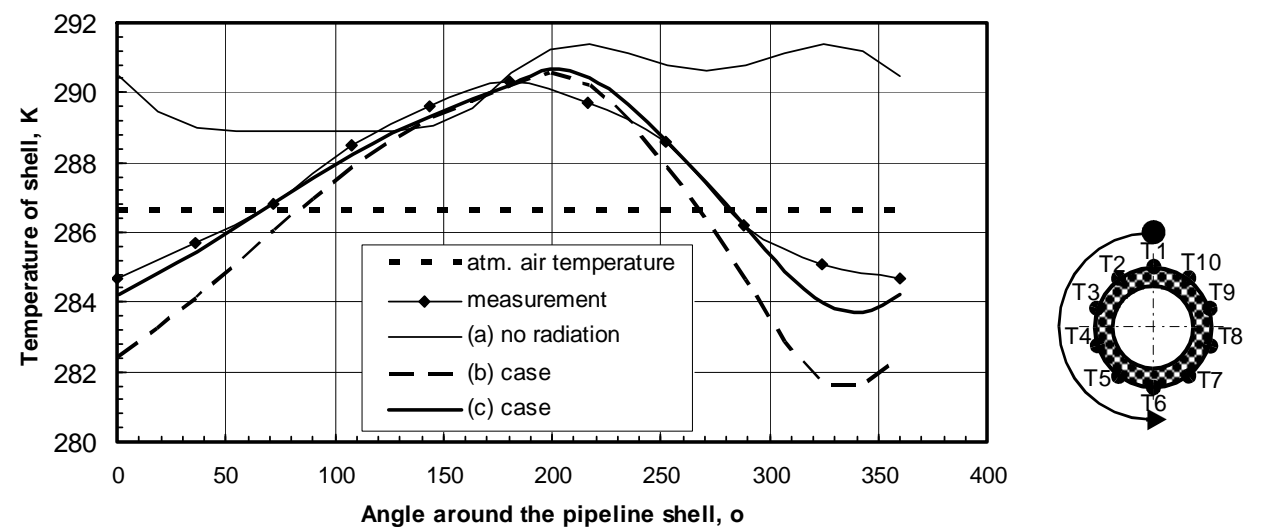

Fig. 12. Temperature distributions of shell around the pipeline, calculation an experimental results,(for atmospheric air temperature $286.9 \mathrm{~K})$, on the right - start point and direction of results collection around the pipeline shell

\section{Final conclusions and remarks}

The exemplary results of measurements carried out at a testing station located in the open space are presented in this paper. The experiments have confirmed that there is a big influence of pipeline shell emissivity on the temperature distribution over its surface. The phenomenon of pipeline shell temperature decrease below air temperature in the case of low temperatures of the sky has been confirmed.

A part of this paper has been devoted to the analysis of radiative properties of atmosphere having the significant influence on the course of radiative heat exchange process between a pipeline and the atmosphere. The calculations for the exemplary parameters of atmospheric air have been done. As the result of these calculations it has been stated that the radiation emission within wavelength $7.5 \div 14 \mu \mathrm{m}$ is of crucial importance in the generation of radiative heat losses in pipelines.

For the chosen case the calculations have been carried out with software Fluent in order to determine theoretical distributions of temperature over the pipeline surface.

The main purpose of the works conducted in that subject is to work out the methods of calculating heat losses and the assessment of the technical condition of thermal insulation in overhead pipelines. This is associated with the elaboration of thermovision measurement of temperature distribution over the surface as well as with the calculations of heat losses.

\section{REFERENCES}

[1] CFD Fluent. User's Guide Fluent 6.3 Documentation. Fluent Inc.

[2] Jeschar R., Kostowski E. and Alt R., „Wärmestrahlung“, Internat. Studies in Science and Engineering, Technische Universität Clausthal, Germany, Politechnika Śląska, Gliwice, Poland, 2004.

[3] Kruczek T., "Determination of radiative ambient temperature during measurements in open air space", Measurement Automation and Monitoring, Vol. 55, No 11, 2009, pp. 882-885, (in Polish).

[4] Kruczek T., "Investigations on determination of heat losses from overhead heat pipelines with application of infrared thermography", Acta Metallurgica Slovaca, No 1, 2009, pp.120-126.

[5] Madany A., "Physics of atmosphere", Oficyna Wyd. Politechniki Warszawskiej, Warszawa, 1996, (in Polish).

[6] Modest M., F., „Radiative heat transfer”, McGraw-Hill, N.York, 2003.

[7] Siegel R., Howell J., R., Thermal radiation heat transfer. 3-rd edition, Hemisphere Publ. Corporation, Washington, 1992.

[8] Wallace J. and Hobbs P., “ Atmospheric Science”, 2-nd edition, Elsevier, 2006.

This work has been performed within the project No NN513 311735 supported financially by the Polish Ministry of Science and Higher Education, 2008-2011 